\title{
Analysis of Binding Energies between Luciferin and Luciferase Adsorbed on Si Surface by Docking Simulations
}

\author{
Katsuhiko Nishiyama $^{\text {a,* }}$, Takanobu Watanabe ${ }^{\mathrm{b}}$, \\ Tadatsugu Hoshino $^{c}$, Iwao Ohdomari ${ }^{\mathrm{b}}$ \\ ${ }^{a}$ Tsuruoka National College of Technology, 104 Sawada, Inooka, Tsuruoka City, \\ Yamagata 997-8511, Japan \\ ${ }^{\mathrm{b}}$ Waseda University, 3-4-1 Ohkubo, Shinjuku-ku, Tokyo 169-8555, Japan \\ ${ }^{\mathrm{c}}$ Chiba University, 1-33 Yayoi-cho, Inage-ku, Chiba 263-8522, Japan
}

\begin{abstract}
The binding energies between luciferin and luciferase adsorbed on a Si surface were estimated by docking simulations. These binding energies were more stable on a hydrophilic Si surface than on a hydrophobic Si surface, but their difference was small. Luciferase adsorbed more strongly on the hydrophobic Si surface than on the hydrophilic Si surface. The luciferase active site was not covered by the Si surface in this adsorption state. The hydrophobic surface would be suitable for the immobilization of luciferase both from the viewpoints of the binding of luciferin to luciferase and the adsorption of luciferase to the Si surface.
\end{abstract}

Key words: luciferase, Si surface, nanostructure, docking simulations, biomedical application, protein chip

PACS: 87.68.+z, code

Preprint submitted to Elsevier Science

16 February 2007

(C) 2007. This manuscript version is made available under the Elsevier user license

http://www.elsevier.com/open-access/userlicense/1.0/ 


\section{Introduction}

The inactivation of enzymes immobilized on solid surfaces is a severe problem for biomedical applications, such as biosensors and bioreactors, because the sensitivity of biosensors and the reaction efficiency of bioreactors are depend on enzymatic activity intensity $[1,2]$. Certain reports have indicated that enzymes are linked on solid surfaces via organic molecules in order to avoid inactivation $[3,4]$. These methods are feasible if these organic molecules do not inhibit enzymatic activities. Current Si-process technology enabled us to fabricate a nanostructure array on a Si surface[5,6]. The delicate control of surface conditions, such as hydrophobic or hydrophilic properties, is also available owing to the recent progress of wet treatment technology for Si. Therefore, a Si surface with a nanofabricated and wet-treated array may be compatible with many types of enzyme. The purposes of our study are to clarify the inactivation mechanism of an enzyme on an artificial surface and to determine surface conditions suitable for immobilization. In our previous study, we analyzed the interactions between luciferase and a Si surface by molecular dynamics (MD) simulation[7]. Results showed that the adsorption-induced changes in the structure of luciferase are larger on the hydrophobic Si surface than on the hydrophilic Si surface. In this study, we analyzed the binding energies between luciferin, the substrate for luciferase, and luciferase adsorbed on the Si surface by docking simulations (Fig. 1). Here, we report the binding energies between luciferin and luciferase adsorbed on the Si surface and surface conditions suitable for the immobilization of luciferase.

\footnotetext{
* Correspoding author. phone number +81-235-25-9076, fax number +81-235-241840
}

Email address: nisiyama@asagi.waseda.jp (Katsuhiko Nishiyama). 


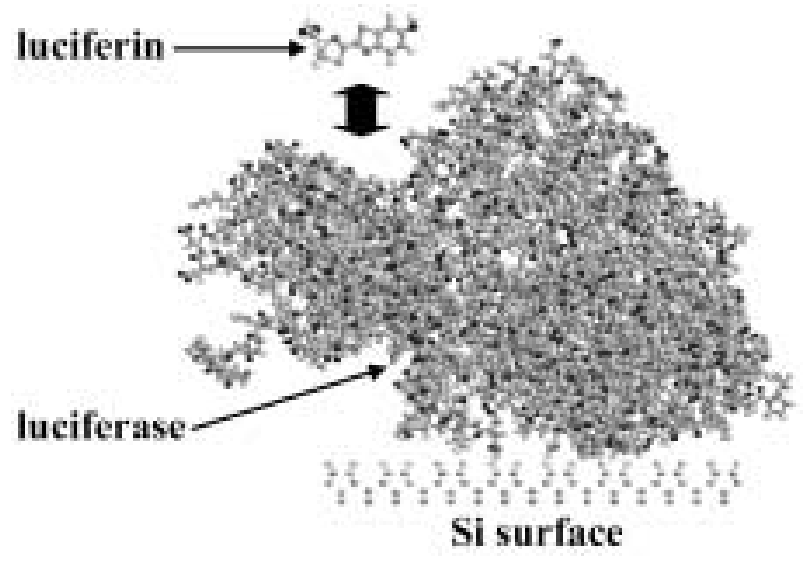

Fig. 1. Binding between luciferin and luciferase adsorbed on Si surface.

\section{Methods}

The docking simulations between luciferin and luciferase were performed using AutoDock3.0[8]. AutoDock3.0 is a suite of programs, making it possible to predict how ligands bind to macromolecules. The Lamarckian genetic algorithm (LGA) was used as a search engine. The step sizes of $0.1 \mathrm{~nm}$ for translation and 50 degrees for rotation were chosen. For each of the 100 independent runs, 10000 LGA operations were generated on a single population of 50 individuals. Operator weights for crossover, mutation, and elitism were set to be $0.80,0.02$, and 1 , respectively. The Solis and Wets algorithm was used as a local search method in the LGA and the contraction and expansion factors were set to be 0.5 and 2.0 , respectively. The structural data for luciferase was derived from our previous MD simulation results[7]. The structural data for luciferin was created on the basis of Hartree-Fock molecular orbital calculations using the program package Gaussian 98[9]. The binding states between luciferin and luciferase were explored within the luciferase active site (residues $218,246,247,251,315,341,342,343,347,348,351$, and 529). Figure 2 shows the luciferase active site. 


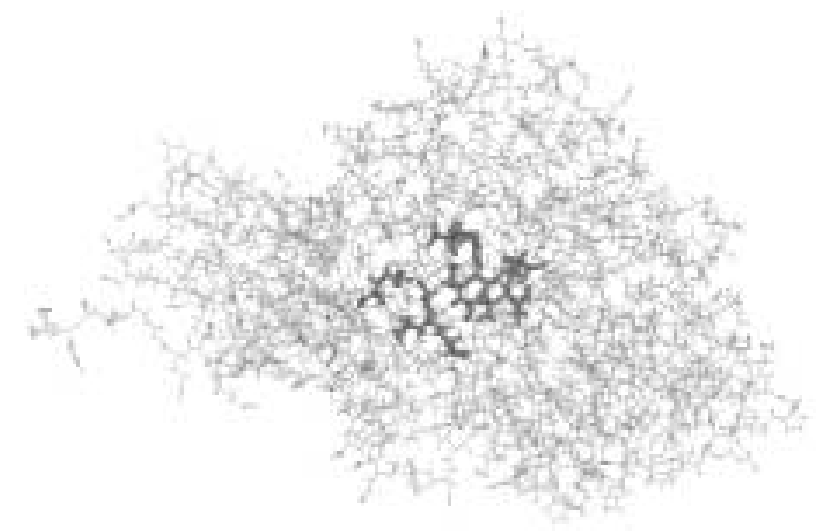

Fig. 2. Luciferase active site (highlighted).

\section{Results and Discussion}

We performed the docking simulations of luciferin to luciferase isolated $_{\text {(luciferase }}$

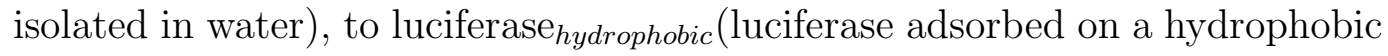
Si surface), and to luciferase ${ }_{\text {hydrophilic }}($ luciferase adsorbed on a hydrophilic $\mathrm{Si}$ surface). Figure 3 shows the binding energies of luciferin to luciferase isolated $_{\text {, }}$ to luciferase hydrophobic $_{\text {, }}$ and to luciferase hydrophilic $_{\text {. }}$

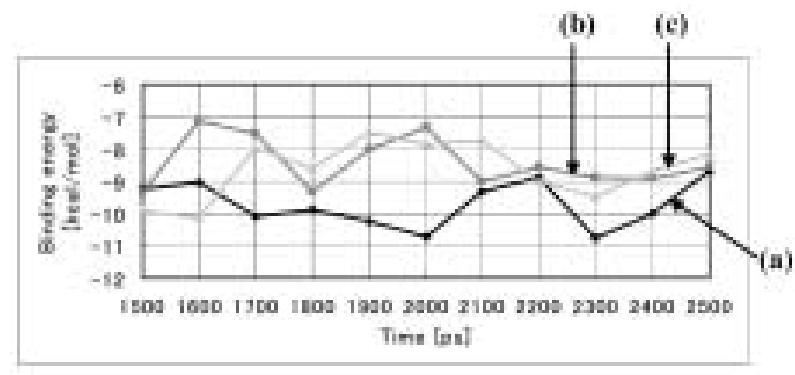

Fig. 3. Binding energies of luciferin to luciferase when isolated (a), adsorbed on hydrophobic surface (b), and adsorbed on hydrophilic surface (c).

The time averages of the binding energies were $-9.71 \mathrm{kcal} / \mathrm{mol}$ for luciferase isolated $_{\text {, }}$ $-8.41 \mathrm{kcal} / \mathrm{mol}$ for luciferase hydrophobic $_{\text {, }}$, and $-8.62 \mathrm{kcal} / \mathrm{mol}$ for luciferase hydrophilic $_{\text {. }}$ The time standard deviations of the binding energies were $0.73 \mathrm{kcal} / \mathrm{mol}$ for luciferase $_{\text {isolated }}, 0.80 \mathrm{kcal} / \mathrm{mol}$ for luciferase hydrophobic $_{\text {, and }} 0.90 \mathrm{kcal} / \mathrm{mol}$ for luciferase $_{\text {hydrophilic }}$. Figure 4 shows the Gaussian distributions of these binding 
energies.

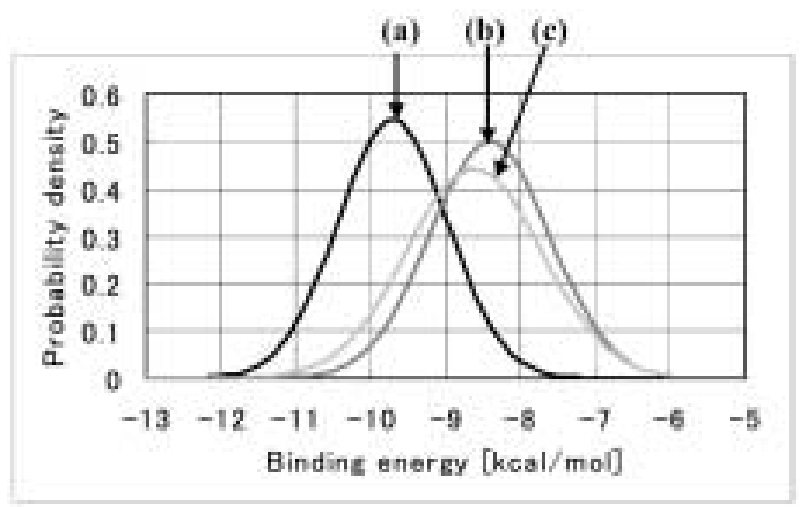

Fig. 4. Gaussian distributions of binding energies of luciferin to luciferase when isolated (a), adsorbed on hydrophobic surface (b), and adsorbed on hydrophilic surface (c).

These results show that the binding energies of luciferin are more stable

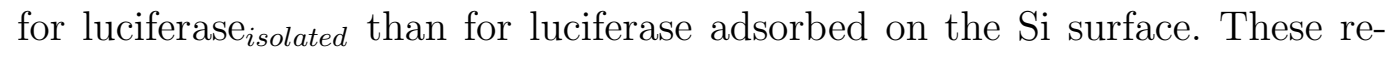
sults also show that the binding energies of luciferin are more stable for

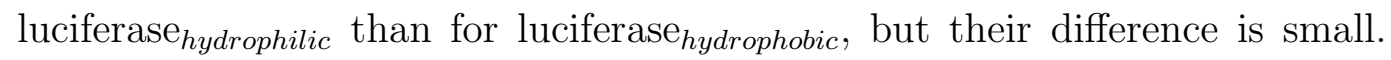
Enzymatic activity should be modified by the deformation of an enzyme active site. Luciferase adsorbed more strongly on the hydrophobic Si surface than on the hydrophilic Si surface[7]. In these adsorption states, a luciferase surface area with a relatively high hydrophobicity contacted the Si surface, and the luciferase active site was not covered by the Si surface[7]. Therefore, the hydrophobic surface would be suitable for the immobilization of luciferase. In a future study, we will perform the docking simulations between luciferin and luciferase adsorbed on the nanofabricated Si surfaces. 


\section{Conclusions}

The binding energies between luciferin and luciferase were more stable on a

hydrophilic Si surface than on a hydrophobic Si surface, but their difference was small. Enzymatic activity should be modified by the deformation of an enzyme active site. Luciferase adsorbed more strongly on the hydrophobic Si surface than on the hydrophilic Si surface. The luciferase active site was not covered by the Si surface in this adsorption state. The hydrophobic surface would be suitable for the immobilization of luciferase both from the viewpoints of the binding of luciferin to luciferase and the adsorption of luciferase to the Si surface.

\section{Acknowledgments}

This study is supported by a Grant-in-Aid for Center of Excellence (COE) Research from the Ministry of Education, Culture, Sports, Science and Technology, by a Grant for the Promotion of the Advancement of Education and Research in Graduate Schools from the Promotion and Mutual Aid Corporation for Private Schools of Japan, and by JST-PRESTO.

\section{References}

[1] T. Tanii, T. Goto, T. Iida, M. K. Masahara and I. Ohdomari, Jpn. J. Appl. Phys. 40, L1135 (2001).

[2] H. Itoh, A. Takahasi, K. Adachi, H. Noji, R. Yasuda, M. Yoshida and K. Kinosita, Jr., Nature 427, 465 (2004). 
[3] Y. S. Lee and M. Mrksich, Trends Biotechnol. 20, s14 (2002).

[4] H. Zhu and M. Snyder, Curr. Opin. Chem. Biol. 7, 55 (2003).

[5] M. Koh, S. Sawara, T. Goto, Y. Ando, T. Shimada and I. Ohdomari, Jpn. J. Appl. Phys. 39, 2186 (2000).

[6] M. Koh, S. Sawara, T. Goto, Y. Ando, T. Shimada and I. Ohdomari, Jpn. J. Appl. Phys. 39, 5352 (2000).

[7] K. Nishiyama, T. Watanabe, T. Hoshino and I. Ohdomari, Jpn. J. Appl. Phys. 45, 1021 (2006).

[8] G. M. Morris, D. S. Goodsell, R. S. Halliday, R. Huey, W. E. Hart, R. K. Belew and A. J. Olson, J. Comput. Chem. 19, 1639 (1998).

[9] M. J. Frisch, G. W. Trucks, H. B. Schlegel, G. E. Scuseria, M. A. Robb, J. R. Cheeseman, V. G. Zakrzewski, J. A. Montgomery, Jr., R. E. Stratmann, J. C. Burant, S. Dapprich, J. M. Millam, A. D. Daniels, K. N. Kudin, M. C. Strain, O. Farkas, J. Tomasi, V. Barone, M. Cossi, R. Cammi, B. Mennucci, C. Pomelli, C. Adamo, S. Clifford, J. Ochterski, G. A. Petersson, P. Y. Ayala, Q. Cui, K. Morokuma, D. K. Malick, A. D. Rabuck, K. Raghavachari, J. B. Foresman, J. Cioslowski, J. V. Qrtiz, B. B. Stefanov, G. Liu, A. Liashenko, P. Piskorz, I. Komaromi, R. Gomperts, R. L. Martin, D. J. Fox, T. Keith, M. A. Al-Laham, C. Y. Peng, A. Nanayakkara, C. Gonzalez, M. Challacombe, P. M. W. Gill, B. G. Johnson, W. Chen, M. W. Wong, J. L. Andres, M. Head-Gordon, E. S. Replogle and J. A. Pople, Gaussian98 revision A.7 (Gaussian Inc., Pittsburgh, PA, 1998). 
${ }^{*}$ Graphical Abstract (pictogram)

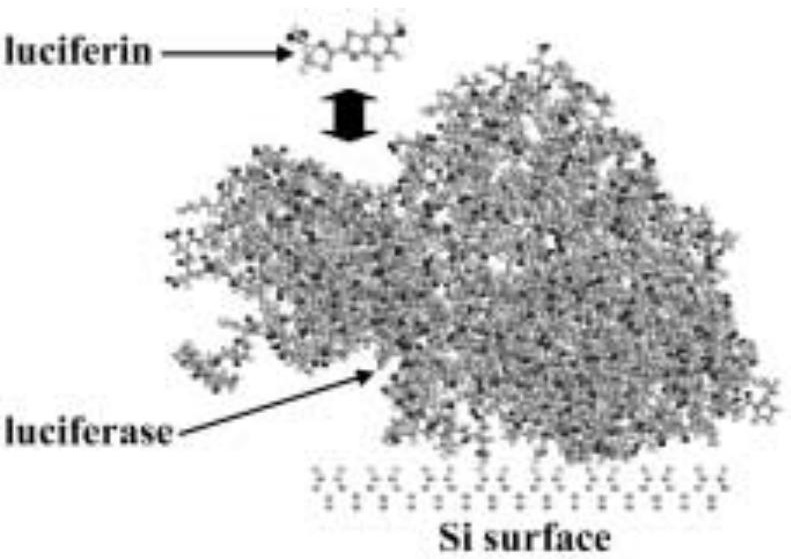




\section{${ }^{*}$ Graphical Abstract (synopsis)}

The binding energies between luciferin and luciferase adsorbed on a si surface were estimated by docking simulations. 